\title{
Study of metamaterial resonators for decoupling of a MIMO-PIFA system
}

\author{
Ignacio Gil* and Raúl Fernández-García \\ Department of Electronic Engineering \\ Universitat Politècnica de Catalunya \\ Colom 1, 08222 Terrassa, Spain \\ *e-mail: ignasi.gil@upc.edu
}

\begin{abstract}
The impact of several subwavelength metamaterial particles based on split-ring inspired resonators to enhance the decoupling level in a two planar inverted-F antenna multi-input multi-output printed circuit board system is investigated. The $\mathrm{S}$ parameters, antenna performance, electric and magnetic field distribution are simulated and analyzed by means of the full 3D electromagnetic simulator CST Microwave Studio at $2.45 \mathrm{GHz}$. The obtained results show a notable coupling reduction level in the three analyzed cases and a trade-off between isolation/antenna performance/area.
\end{abstract}

Keywords-PIFA antennas, electromagnetic coupling, metamaterial.

\section{INTRODUCTION}

Multi-input multi-output (MIMO) antennas technology allows improving the spectral efficiency of wireless communication systems. The signals on the multiple antennas at both the transmitting and receiving front-ends are combined so that the data rate and bit error rate are improved [1]. A MIMO system critical electromagnetic compatibility (EMC) issue consists of optimizing isolation between antennas in order to preserve the system performance. The higher the antenna separation on the printed circuit board (PCB) the better the isolation performance. A typical separation distance of $\lambda / 2$ significantly reduces the coupling level [2]. However, there is a trade-off with regard to the integration level in spaceconstrained wireless terminals.

Recently, many research works have been focused on reducing the electromagnetic coupling between MIMO antennas. In [3] a scheme of a pair of resonating coupling slits on the common ground plane between antennas is presented. A uniplanar solution between antennas is presented in [4]. Another method based on an open-ended ground T-shaped slot is reported in [5]. Other papers have applied metamaterial structures, such as split rectangular loop resonators, between two individual radiating elements to reduce their mutual coupling [6,7]. Alternatively, as demonstrated in [8] by the authors, sub-wavelength resonators based on split-ring resonators (SRRs) are able to improve the electromagnetic compatibility of a planar inverted-F antenna (PIFA) with nearby conducting elements by reducing the coupling level.

In this paper, an expansion of the previous research is developed in order to study the impact of several SRR inspired metamaterial resonators in decreasing the coupling impact of a MIMO PIFA system (Fig. 1) operating at applications included

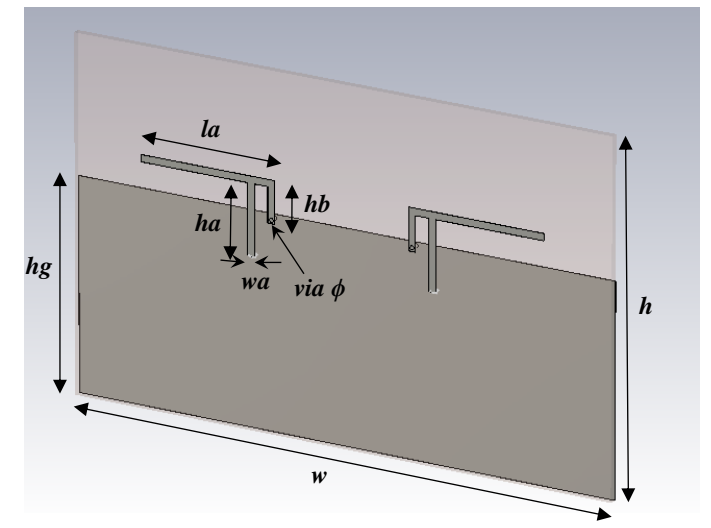

Fig. 1. Geometry of the 2-element MIMO system based PIFA antennas.

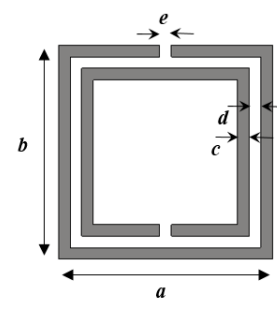

(a)

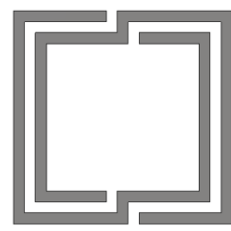

(b)

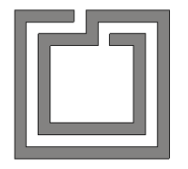

Fig. 2. Considered metamaterial particles. (a) SRR, (b) NB SRR and (c) $\mathrm{SR}$, with their corresponding dimensions.

in the $2.45 \mathrm{GHz}$ industrial, scientific and medical (ISM) radio band. Specifically, the SRR, the nonbianisotropic split-ring resonator (NB SRR), and spiral resonator (SR) [9] have been considered as subwavelength resonators (Fig. 2). The study has been carried out by means of full-3D electromagnetic simulations performed with the commercial software CST Microwave Studio.

The paper is organized as follows. In Section II the analysis of the electromagnetic fields is carried out in order to identify the maximum flow of electromagnetic energy produced by the mutual coupling between PIFA antennas. In Section III three metamaterial particles (SRR, NB SRR and SR) are analyzed and compared with the aim to enhance the decoupling level in the MIMO system. Finally, the main conclusions are drawn and summarized in Section IV. 


\section{MIMO INVESTIGATION OF ELECTROMAGNETIC FIELD DISTRIBUTION}

PIFA is a common antenna for portable devices because of its excellent balance between low-profile, low-cost and performance. This antenna behaves like a $\lambda / 4$ monopole printed on circuit boards, presenting a ground point and a feed point along the main resonant structure. Typical PIFA performance consists of bandwidth $\geq 100 \mathrm{MHz}, \quad \mathrm{VSWR} \leq 2.5 \quad$ and efficiency $\geq 60 \%$.

The distance between the PIFA antennas has been selected at $\lambda / 6$ in order to compact the two-element MIMO antenna and to allow the placement of the proposed subwavelength metamaterial resonators. A low-cost commercial RO3003 substrate (dielectric constant $\varepsilon_{\mathrm{r}}=3$, thickness $h=1.52 \mathrm{~mm}$ ) has been used. The dimensions of the $50 \Omega$ transmission line feed structure without an isolation mechanism is reported in Fig.1: $h=50 \mathrm{~mm} ; w=80 \mathrm{~mm} ; h g=30 \mathrm{~mm} ; h a=10 \mathrm{~mm} ; h b=6 \mathrm{~mm}$; $l a=20 \mathrm{~mm} ; w a=1 \mathrm{~mm}$; via $\phi=0.6 \mathrm{~mm}$; antenna separation=20 $\mathrm{mm}$.

The return loss (S11) and insertion loss (coupling level, S21) from one PIFA antenna to the other one are depicted in Fig. 3. The antennas operate properly at $2.45 \mathrm{GHz}$ (since $\mathrm{S} 11<-$ $10 \mathrm{~dB}$ ) and the maximum coupling level corresponds to $\mathrm{S} 21=-9$ $\mathrm{dB}$. The reference antenna gain and efficiency correspond to: $\mathrm{G}=1.7 \mathrm{dBi} ; \eta=82 \%$. Two mechanisms are responsible of the coupling level between antennas: the coupling through the air and the substrate modes. To analyze both mechanisms, a single port excitation is assumed (left antenna) and the electric and magnetic field magnitude are investigated on the surface of the PCB (Figs. 4 and 5). The coupling mechanism is observed in both cases according to the field excitation of the right nonexcited antenna. The electric field is significant in the vicinity of both antennas and reaches a minimum in the center of the PCB. Alternatively, the magnetic field is significant in the area located between both antennas, suggesting the presence of substrate modes. In any case, the available area between PIFA antennas can be used to place the metamaterial subwavelength particles in order to reduce the coupling mechanism and preserve the MIMO performance.

\section{ELECTROMAGNETIC DECOUPLING DESIGN PROCESS AND DISCUSSION}

SRRs and their derived particles are considered narrowband frequency resonators. Therefore, a narrowband coupling reduction is expected. Fundamentally, SRRs behave as LC tanks and resonate by an external magnetic flux, but they also can be excited by a properly polarized time-varying electric field, due to cross-polarization effects. Those effects are not produced in the NB SRRs because of the $180^{\circ}$ rotation symmetry in the plane of the particle. Anyway the LC equivalent circuit is identical with regard to the SRR, as well as the resonance frequency and occupied area. Alternatively, the SRs present another tank resonator behavior but according to the equation (1). As a consequence, its area can be roughly reduced in a factor 2 with regard to the conventional SRR.

$$
f_{S R R}=\frac{1}{2 \pi \sqrt{L C}} \approx f_{N B S R R} \approx 2 f_{S R}=\frac{1}{\pi \sqrt{L C}}
$$

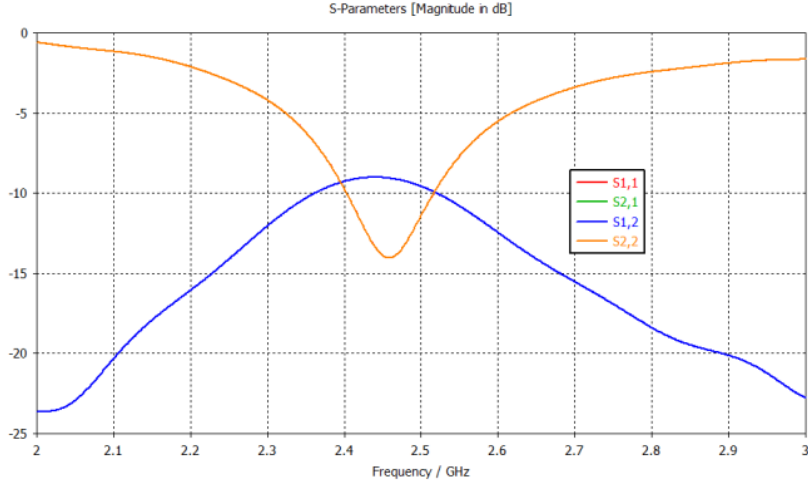

Fig. 3. S-parameters of the initial configuration of the MIMO antennas.

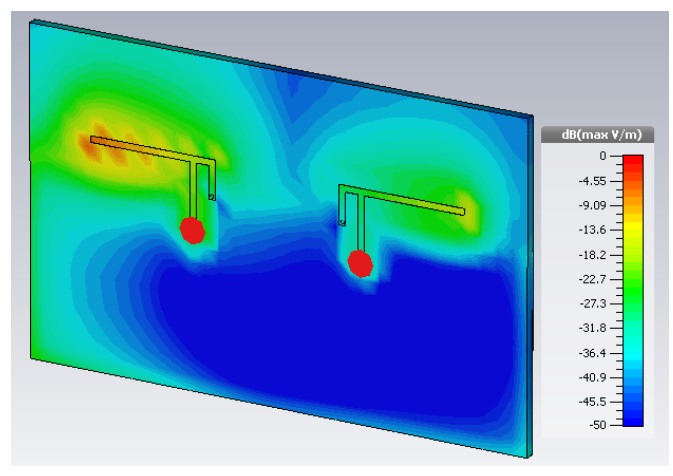

Fig. 4. Normalized electric field magnitude $(2.45 \mathrm{GHz})$ at the horizontal plane on the top of the substrate of the MIMO structure.

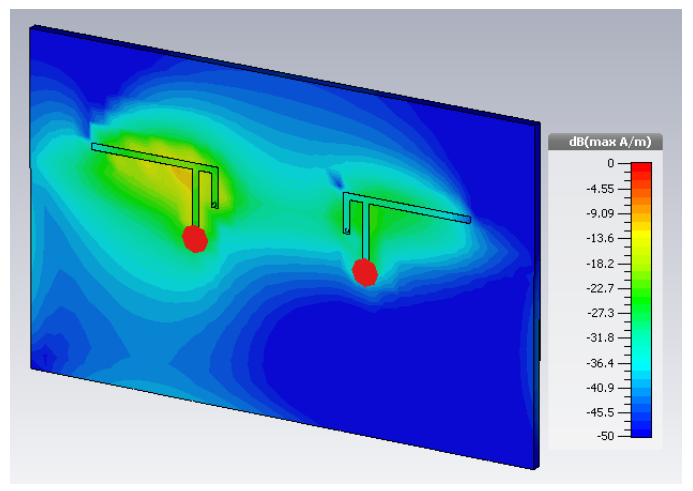

Fig. 5. Normalized magnetic field magnitude $(2.45 \mathrm{GHz})$ at the horizontal plane on the top of the substrate of the MIMO structure.

\section{A. Split-ring resonator (SRR)}

The $\mathrm{p}$ SRR has been designed and tailored by means of the electromagnetic simulator in order to resonate at 2.45 $\mathrm{GHz}$. The location of the particle has been sweeped to achieve the optimized decoupling level. The final SRR is etched on the top of the substrate (antenna level) and its dimensions are: $a=b=7.4 \mathrm{~mm} ; c=d=e=0.4 \mathrm{~mm}$, corresponding to a compact subwavelength particle area of $0.061 \lambda$ x $0.061 \lambda$. Fig. 6 shows the obtained S-parameters. The symmetry of the particle guarantees $\mathrm{S} 11=\mathrm{S} 22$ and $\mathrm{S} 12=\mathrm{S} 21$. The main result 


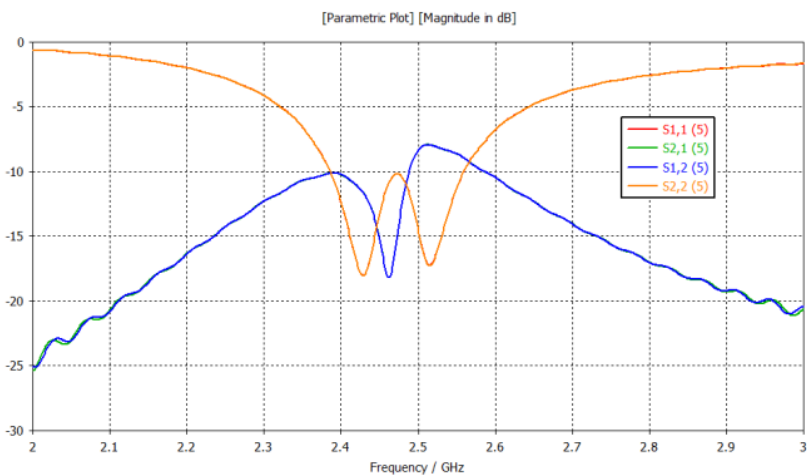

Fig. 6. S-parameters of the MIMO+SRR proposed structure.

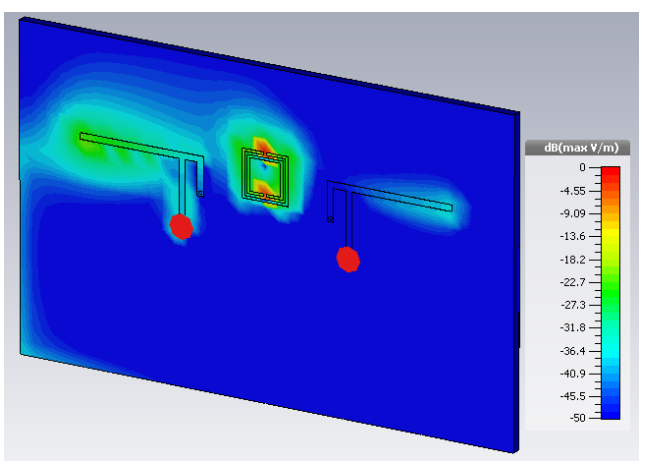

Fig. 7. Normalized electric field magnitude $(2.45 \mathrm{GHz})$ at the horizontal plane on the top of the substrate of the MIMO+SRR structure.

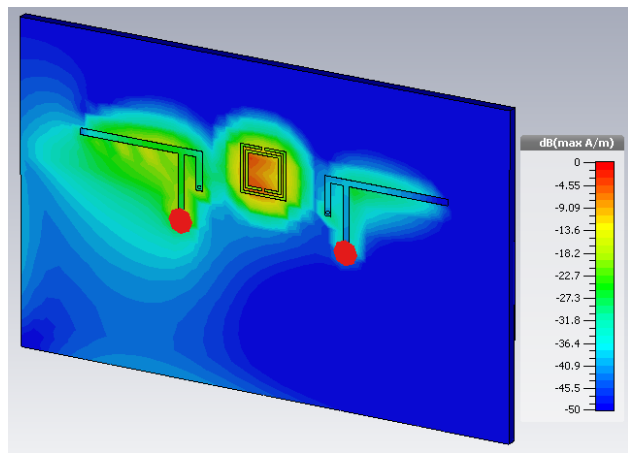

Fig. 8. Normalized magnetic field magnitude $(2.45 \mathrm{GHz})$ at the horizontal plane on the top of the substrate of the MIMO+SRR structure.

corresponds to the significant coupling reduction up to $\mathrm{S} 21=-$ $17.9 \mathrm{~dB}$ at the operation frequency. Moreover, the antenna performance is still acceptable since the criterion $\mathrm{S} 11<-10 \mathrm{~dB}$ is satisfied. In addition the antenna parameters are increased with regard to the initial case: $\mathrm{G}=3.3 \mathrm{dBi} ; \eta=90.7 \%$, suggesting a radiation effect produced by the SRR presence. Figs. 7 and 8 illustrate the electric and magnetic field distribution across the surface of the MIMO PCB including the designed SRR. In both cases a clear reduction of the corresponding field in the non-excited PIFA is observed. Note that the SRR is excited at the operation frequency. An increase of the electric field is observed in the edges of the particle whereas the central area is mainly excited by the magnetic

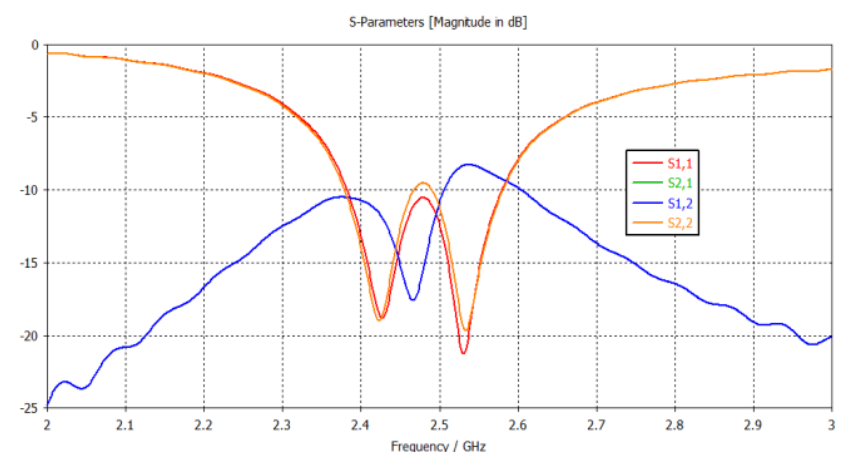

Fig. 9. S-parameters of the MIMO+NB SRR proposed structure.

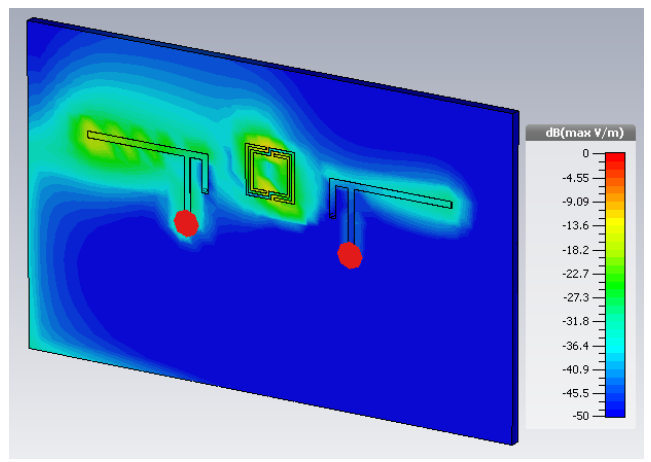

Fig. 10. Normalized electric field magnitude $(2.45 \mathrm{GHz})$ at the horizontal plane on the top of the substrate of the MIMO+NB SRR structure.

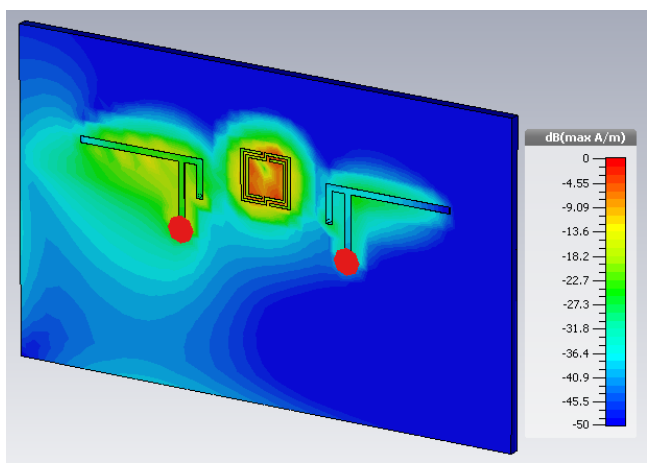

Fig. 11. Normalized magnetic field magnitude $(2.45 \mathrm{GHz})$ at the horizontal plane on the top of the substrate of the MIMO+NB SRR structure.

field, as expected due to its intrinsic resonant magnetic dipole nature.

\section{B. Non-bianosotropic split-ring resonator (NB SRR)}

A similar design procedure has been followed in order to determine the NB SRR dimensions and position on the top of the substrate. The final resonator dimensions are: $a=8 \mathrm{~mm}$; $b=7.8 \mathrm{~mm} ; c=d=e=0.4 \mathrm{~mm}$, corresponding to a compact subwavelength particle area of $0.066 \lambda \times 0.064 \lambda$. In this case, a slight difference is produced between S-parameters (Fig. 9) due to the subtle asymmetry degree of the NB SRR topology. The simulated coupling level is $\mathrm{S} 21=-15.2 \mathrm{~dB}$ at the operation frequency. Again, the antenna performance is still acceptable since the criterion $\mathrm{S} 11<-10 \mathrm{~dB}$ is satisfied and the antenna 


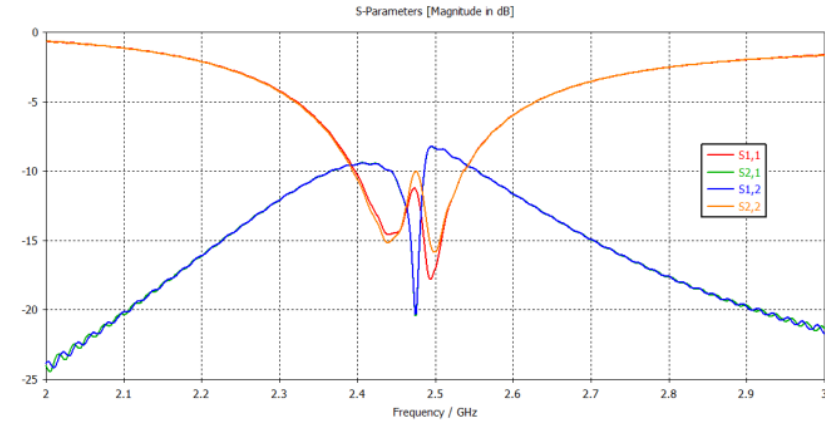

Fig. 12. S-parameters of the MIMO+SR proposed structure.

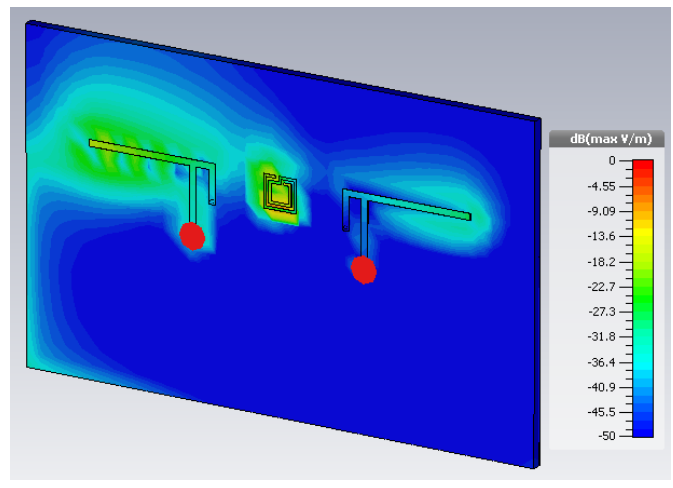

Fig. 13. Normalized electric field magnitude $(2.45 \mathrm{GHz})$ at the horizontal plane on the top of the substrate of the MIMO+SR structure.

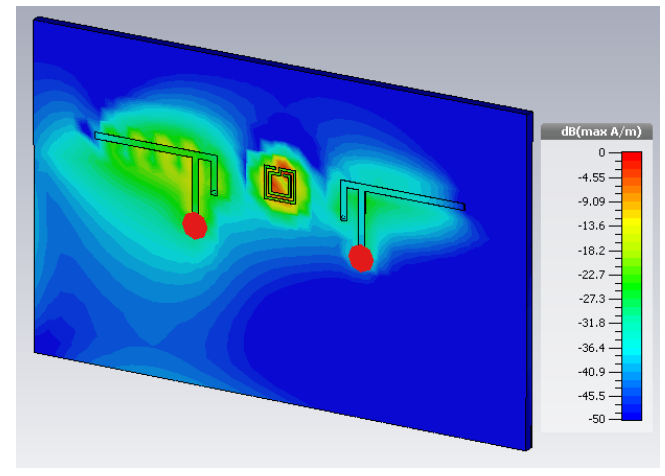

Fig. 14. Normalized magnetic field magnitude $(2.45 \mathrm{GHz})$ at the horizontal plane on the top of the substrate of the MIMO+SR structure.

parameters remain roughly constant, in comparison with the SRR case: $\mathrm{G}=3.5 \mathrm{dBi} ; \eta=91.5 \%$. Figs. 10 and 11 illustrate the electric and magnetic field distribution across the surface of the MIMO PCB including the designed NB SRR. In this case, the particle effectiveness against the electric coupling is significantly lower with regard to the previous study case due to the particle geometry, as expected. This fact explains the better reduction in the S21 parameter from the SRR topology. Nevertheless, the magnetic decoupling is similar than the corresponding to the SRR case. As in the previous case, the NB $\mathrm{SRR}$ is clearly excited at the operation frequency.

\section{Spiral resonator $(S R)$}

The last study case corresponds to the SR. As explained previously, theoretically, a factor 2 area reduction is expected in order to operate at $2.45 \mathrm{GHz}$ with regard to the previous cases. Indeed, the proposed SR dimensions correspond to: $a=5.2 \mathrm{~mm} ; b=5 \mathrm{~mm} ; c=d=e=0.4 \mathrm{~mm}$ and therefore an ultracompact metamaterial area of $0.043 \lambda \times 0.041 \lambda$ is achieved. The asymmetry of the particle explains the slight difference observed in S-parameters (Fig. 12). The simulated coupling level is $\mathrm{S} 21=-19.6 \mathrm{~dB}$ which corresponds to the maximum value in comparison with their counterpart particles. However, in this case the decoupling bandwidth is clearly decreased. The antenna performance is acceptable, even though the gain and efficiency are decreased due to the lower area radiation of the $\mathrm{SR}(\mathrm{S} 11<-10 \mathrm{~dB} ; \mathrm{G}=2.2 \mathrm{dBi} ; \eta=86.4 \%)$. Figs. 13 and 14 illustrate the electric and magnetic field distribution of the MIMO and SR design. Both, electric and magnetic decoupling is produced with regard to the reference antenna case at the operation frequency.

Table I summarizes the obtained results for all the studied cases.

TABLE I. MIMO ANTENNA PERFORMANCE AND COUPLING REDUCTION WITH METAMATERIAL PARTICLES @ $2.45 \mathrm{GHZ}$

\begin{tabular}{|c|c|c|c|c|c|}
\hline $\begin{array}{c}\text { Topology/ } \\
\text { Performance }\end{array}$ & $\begin{array}{c}\text { PIFA } \\
\text { Gain } \\
(\mathbf{d B i})\end{array}$ & $\begin{array}{c}\text { PIFA } \\
\text { Eff. } \\
(\%)\end{array}$ & $\begin{array}{c}\text { PIFA } \\
\text { S11 } \\
(\mathbf{d B})\end{array}$ & $\begin{array}{c}\text { Coupling } \\
\text { S21 } \\
(\mathbf{d B})\end{array}$ & $\begin{array}{c}\text { Coupling } \\
\text { reduction } \\
(\%)\end{array}$ \\
\hline$M I M O$ & 1.7 & 82.0 & -13.9 & -9.0 & - \\
\hline$M I M O+S R R$ & 3.3 & 90.7 & -13.2 & -17.9 & 98.9 \\
\hline$M I M O+N B S R R$ & 3.5 & 91.5 & -13.8 & -15.2 & 68.9 \\
\hline$M I M O+S R$ & 2.2 & 86.4 & -14.4 & -19.6 & 117.8 \\
\hline
\end{tabular}

\section{CONCLUSION}

In this work, the isolation improvement of a MIMO PIFA system based on several SRR family metamaterial particles has been studied. The technique relies on the effectiveness of the proposed subwavelength resonators to reduce the mutual coupling between the PIFA antennas. SRR, NB SRR and SR have been designed to resonate at the antenna operation frequency and located in the optimum place in order to maximize the electric and magnetic field decoupling. The full 3D electromagnetic simulation results show a notable coupling reduction level in the three analyzed cases. A trade-off between isolation/antenna performance/area is concluded. SRR allows obtaining a good compromise between the three criteria. The best isolation case in terms of absolute magnitude corresponds to the SR but with a clear reduction in the decoupling bandwidth. The optimum case in terms of antenna gain and efficiency correspond to the NB SRR but with a minimum decoupling effect. Some preliminary experimental measurements have demonstrated the effectiveness of the presented results and the final test bench and samples are in progress to validate the CST Microwave Studio simulation results. 


\section{REFERENCES}

[1] Y. Huang, and K. Boyle, "Antennas from theory to practice", John Wiley \& Sons Inc., 2008.

[2] H. Carrasco, H. D. Histov, R. Feick, and D. Cofr, "Mutual coupling between planar inverted-F antennas", Microw. Opt. Technol. Letters, vol. 42, pp. 224-227, August 2004.

[3] T. Kokkinos, E. Liakou and A. P. Feresidis, "Decoupling antenna elements of PIFA arrays on handheld devices", Electronics Letters, vol. 44, pp. 1442-1444, December 2008.

[4] S.D. Assimonis, T. V. Yioultsis, M. Hinaje, and C. S. Antonopoulos, "Mutual coupling reduction in MIMO antennas at $5.6 \mathrm{GHz}$ (WiMAX) vased on a proposed uniplanar easily fabricated structure", IEEE International Symposium on Electromagnetic Compatibility, pp. 1-4, Rome (Italy), September 2012.

[5] J. Park, J. Choi, J.-Y. Park, and Y.-S. Kim, "Study os a T-shaped slot with a capacitor for high isolation between MIMO antennas", IEEE Antennas and Wireless Propagation Letters, vol. 11, pp. 1541-1544, October 2012.

[6] C.-C. Hsu, K.-H. Lin and H.-L. Su, "Implementation of broadband isolator using metamaterial-inspired resonators and a T-shaped branch for MIMO antennas", IEEE Transactions on Antennas and Propagation, vol. 59, pp. 3936-3939, October 2011.

[7] D. A. Ketzaki, and T. V. Yioultsis, "Metamaterial-based design of planar compact MIMO monopoles", IEEE Transactions on Antennas and Propagation, vol. 61, pp. 2758-2766, May 2013.

[8] I. Gil, and R. Fernández-García, "Analysis of PIFA antenna coupling in nearby traces and reduction with CSRRs in PCB at $2.45 \mathrm{GHz}$ ", IEEE International Symposium on Electromagnetic Compatibility, pp. 361365, Dresden (Germany), August 2015.

[9] J.D. Baena, J. Bonache, F. Martín, R. Marqués, F. Falcone, T. Lopetegui, M.A.G. Laso, J. García-García, I. Gil, M. Flores Portillo and M. Sorolla. "Equivalent-circuit models for split-ring resonators and complementary split-ring resonators coupled to planar transmission lines", IEEE Transactions on Microwave Theory and Techniques, vol. 53, pp. 1451-1461, April 2005. 